\title{
Solute partitioning in polymer-salt ATPS: The Collander equation
}

\author{
Sara C. Silvério ${ }^{\mathrm{a}, \mathrm{b}}$, Oscar Rodriguez ${ }^{\mathrm{a}}$, José A. Teixeira ${ }^{\mathrm{b}}$, Eugénia A. Macedo ${ }^{\mathrm{a}, *}$ \\ a LSRE/LCM-Laboratory of Separation and Reaction Engineering, Faculdade de Engenharia da Universidade do Porto, Rua Dr. Roberto Frias, $4200-465$ Porto, Portugal \\ ${ }^{\mathrm{b}}$ IBB-Institute for Biotechnology and Bioengineering, Centre of Biological Engineering, Universidade do Minho, Campus de Gualtar, 4710-057 Braga, Portugal
}

\section{A R T I C L E I N F O}

\section{Article history:}

Received 18 January 2010

Received in revised form 14 April 2010

Accepted 19 April 2010

Available online 24 April 2010

\section{Keywords:}

Aqueous two-phase system

Polymer-salt

Collander equation

Proteins

\begin{abstract}
A B S T R A C T
The partition coefficients for several solutes (five nitrophenylated-monosaccharides and four proteins) were experimentally determined, at $23^{\circ} \mathrm{C}$, in three different tie-lines of two polymer-salt aqueous twophase systems (ATPS): UCON- $\mathrm{K}_{2} \mathrm{HPO}_{4}$ and $\mathrm{UCON}-\mathrm{NaH}_{2} \mathrm{PO}_{4}$. These partition coefficients together with others obtained from the literature for five dinitrophenylated-amino acids were used to investigate the suitability of the Collander equation to correlate partition coefficients in polymer-salt ATPS. This equation was first proposed to describe the linear correlation between partition coefficients of solutes in different water-organic solvent systems. More recently, it was proved that partition coefficients for several biomolecules in polymer-polymer ATPS can also be correlated with this equation. In this work, several correlations were tested: partition coefficients obtained for different tie-lines within the same system and also partition coefficients obtained from different systems. In both cases, a linear relation was observed, despite a less satisfactory correlation was found when different ATPS were compared. Overall, it was demonstrated that the Collander equation can be used to satisfactorily correlate solute partitioning in the studied polymer-salt ATPS.
\end{abstract}

(c) 2010 Elsevier B.V. All rights reserved.

\section{Introduction}

Aqueous two-phase systems (ATPS) are biphasic systems composed mainly by water. This kind of systems can be obtained combining aqueous solutions of different compounds (two polymers, a polymer and a salt, or other alternative components) that become partially immiscible above some critical conditions like concentration or temperature. ATPS have been successfully applied for the separation of different kinds of solutes, such as metal ions [1,2], proteins [3,4], DNA and RNA [5], cells [6] or viruses [3].

The Collander equation was first developed in 1947 for partitioning in (water + organic solvent) systems [7]. In his original work, R. Collander observed that partition coefficients of several organic solutes in a given organic solvent/water binary system plotted against the partition coefficients of the same solutes in a different organic solvent/water system gave a straight line when using a logarithmic scale. Therefore, the following linear equation was obtained:

$\ln K_{1}=a \cdot \ln K_{2}+b$

where $K_{1}$ and $K_{2}$ represent the partition coefficients in the systems 1 and 2, respectively, and $a$ and $b$ are two empirical constants. The Collander equation assumes a linear relation between the log-

\footnotetext{
* Corresponding author. Tel.: +35122508 1653.

E-mail address: eamacedo@fe.up.pt (E.A. Macedo).
}

arithms of the partition coefficient of solutes in different biphasic systems. During the years this equation has been used in many different situations [8-10]. Recently, the partition coefficients for several biomolecules in different polymer-polymer ATPS were successfully correlated according to the Collander equation [11,12].

In this work the linear correlation described by the Colander equation was investigated in polymer-salt ATPS. For this purpose, the partition coefficients of several solutes (five nitrophenylatedmonosaccharides, NP-monosaccharides, and four proteins) were experimentally determined in three different tie-lines of two biphasic systems: $\mathrm{UCON}-\mathrm{K}_{2} \mathrm{HPO}_{4}$ and $\mathrm{UCON}-\mathrm{NaH}_{2} \mathrm{PO}_{4}$. These experimental data were completed with data from the literature for 5 dinitrophenylated-amino acids (DNP-amino acids) previously reported for the same ATPS. The polymer used, UCON, belongs to the group of the so-called thermo-separating polymers. When heated above the critical temperature (about $50^{\circ} \mathrm{C}$ ) UCON separates from the aqueous solution, thus facilitating polymer recovery and reutilization $[13,14]$.

\section{Experimental}

\subsection{Materials}

UCON 50-HB-5100, a random copolymer (average molecular weight $\mathrm{Mr}=3900$ ) of $50 \%$ ethylene oxide and $50 \%$ propylene oxide, was obtained from Union Carbide (NY, USA). Di-potassium hydrogen phosphate $\left(\mathrm{K}_{2} \mathrm{HPO}_{4}\right)$ (anhydrous, 99.99 Suprapur) and sodium 
Table 1

Partition coefficients obtained for the several solutes in the UCON-K $\mathrm{HPO}_{4}$ and UCON-NaH $\mathrm{PO}_{4}$ ATPS

\begin{tabular}{|c|c|c|c|}
\hline \multicolumn{4}{|l|}{$\mathrm{UCON}-\mathrm{K}_{2} \mathrm{HPO}_{4}$} \\
\hline Solute & Tie-line A (18.02\% Polymer-3.8\% Salt) & Tie-line B (19.98\% Polymer-4\% Salt) & Tie-line C (22\% Polymer-4.2\% Salt) \\
\hline NP- $\alpha$-Gluco & $2.166 \pm 0.059$ & $2.553 \pm 0.034$ & $3.025 \pm 0.047$ \\
\hline NP- $\beta$-Gluco & $2.288 \pm 0.051$ & $2.629 \pm 0.045$ & $3.107 \pm 0.057$ \\
\hline NP- $\beta$-Galacto & $2.024 \pm 0.037$ & $2.269 \pm 0.031$ & $2.631 \pm 0.038$ \\
\hline NP- $\alpha-M a n n o$ & $2.433 \pm 0.030$ & $2.925 \pm 0.043$ & $3.523 \pm 0.051$ \\
\hline NP- $\beta$-Fuco & $2.588 \pm 0.033$ & $3.199 \pm 0.064$ & $3.581 \pm 0.043$ \\
\hline BSA & $0.01920 \pm 0.00090$ & $0.01468 \pm 0.00072$ & $0.01276 \pm 0.00076$ \\
\hline $\mathrm{Hb}$ & $0.0295 \pm 0.0011$ & $0.0284 \pm 0.0018$ & $0.0249 \pm 0.0012$ \\
\hline Myb & $0.0383 \pm 0.0025$ & $0.0216 \pm 0.0013$ & $0.02180 \pm 0.00068$ \\
\hline ChymA & $0.0485 \pm 0.0029$ & $0.0373 \pm 0.0022$ & $0.03504 \pm 0.00076$ \\
\hline \multicolumn{4}{|c|}{ UCON-NaH ${ }_{2} \mathrm{PO}_{4}$} \\
\hline Solute & Tie-line A ( $16 \%$ polymer-6.5\% salt) & Tie-line B ( $18.26 \%$ polymer-6.99\% salt) & Tie-line C ( $20 \%$ polymer- $8 \%$ salt $)$ \\
\hline NP- $\alpha$-Gluco & $3.513 \pm 0.035$ & $4.651 \pm 0.069$ & $6.373 \pm 0.067$ \\
\hline NP- $\beta$-Gluco & $3.708 \pm 0.045$ & $4.957 \pm 0.091$ & $7.09 \pm 0.12$ \\
\hline$N P-\beta$-Galacto & $3.057 \pm 0.039$ & $3.995 \pm 0.090$ & $5.269 \pm 0.099$ \\
\hline NP- $\alpha-M a n n o$ & $4.625 \pm 0.086$ & $5.839 \pm 0.052$ & $7.96 \pm 0.15$ \\
\hline NP- $\beta$-Fuco & $4.426 \pm 0.059$ & $5.409 \pm 0.068$ & $7.355 \pm 0.053$ \\
\hline BSA & $0.0365 \pm 0.0018$ & $0.02800 \pm 0.00056$ & $0.01756 \pm 0.00060$ \\
\hline $\mathrm{Hb}$ & $0.0635 \pm 0.0015$ & $0.0796 \pm 0.0048$ & - \\
\hline Myb & $0.0656 \pm 0.0034$ & $0.0606 \pm 0.0042$ & $0.0489 \pm 0.0026$ \\
\hline ChymA & $0.1229 \pm 0.0029$ & $0.1160 \pm 0.0038$ & $0.0753 \pm 0.0028$ \\
\hline
\end{tabular}

dihydrogen phosphate dihydrate $\left(\mathrm{NaH}_{2} \mathrm{PO}_{4} \cdot 2 \mathrm{H}_{2} \mathrm{O}\right)$ (p.a., Reag. $\mathrm{Ph}$ Eur) were supplied by Merck. Stock solutions of each chemical were prepared in deionised water (ca. $50 \mathrm{wt} \%$ for UCON, $20 \mathrm{wt} \%$ for $\mathrm{K}_{2} \mathrm{HPO}_{4}$ and for $\mathrm{NaH}_{2} \mathrm{PO}_{4}$ ) and all concentrations were obtained gravimetrically after evaporation on heating plate (Stuart hotplate SB300) for salts or after lyophilization (Scan Vac, model CoolSafe 55-4) for UCON.

NP-monosaccharides: 4-nitrophenyl- $\beta$-D-glucopyranoside, 4nitrophenyl- $\beta$-D-galactopyranoside, 4 -nitrophenyl- $\alpha$-D-mannopyranoside and 4-nitrophenyl- $\beta$-D-fucopyranoside were obtained from USB and 4-nitrophenyl- $\alpha$-D-glucopyranoside was obtained from Sigma. Stock solutions of the five NP-monosaccharides were prepared in deionised water (0.2 wt\%).

Proteins: bovine serum albumin (BSA), bovine hemoglobin $(\mathrm{Hb})$, horse myoglobin (Myb) and bovine $\alpha$-chymotrypsinogen $\mathrm{A}$ (ChymA) were obtained from Sigma. Stock solutions of the four proteins were prepared in deionised water (ca. $0.4 \mathrm{wt} \%)$.

BIOQUANT protein reagent solution for the Bradford method was supplied by Merck.

All products were used as received without further purification. Deionised water was used for all diluting purposes. All weighing was carried out on an Adam Equipment balance model AAA250L, precise to within $\pm 0.2 \mathrm{mg}$.

\subsection{Methods}

The procedure to obtain the partition coefficients has been explained in detail before $[15,16]$. Suitable amounts of a stock solution of each solute (from 0 to $100 \mathrm{mg}$ ) were added to six replicates of the ATPS with the same feed composition. The corresponding amount of water (ranging from 100 to $0 \mathrm{mg}$ ) was also added to keep all compositions constant except for the solutes. The components were thoroughly vortex-mixed (VWR, model VV3) for 2 min and then centrifuged (minispin, Eppendorf) at $10^{4}$ r.p.m. for $15 \mathrm{~min}$ to accelerate phase separation. Solute quantification in the top and bottom phases was achieved using different methodologies. For NP-monosaccharides, samples of each phase were withdrawn, conveniently diluted with water, and their absorbance measured at $302 \mathrm{~nm}$ in a UV-vis spectrophotometer (Thermo electron corporation, UV1). Protein quantification was obtained using the Bradford method. In this case, samples from the top and bottom phase were withdrawn, conveniently diluted with water and mixed with the Bradford reagent. After 2 min reaction, their absorbance was measured at $595 \mathrm{~nm}$. Partition coefficients were determined as the slope of the straight line obtained when comparing the absorbance in the top phase against that in the bottom phase, corrected with the corresponding dilution factors (DF):

$K=\frac{\mathrm{Abs}(\mathrm{top}) \cdot \mathrm{DF}_{\mathrm{top}}}{\mathrm{Abs}(\text { bottom }) \cdot \mathrm{DF}_{\mathrm{bottom}}}$

\section{Results and discussion}

Six different UCON-phosphate salt ATPS were prepared: three tie-lines of $\mathrm{UCON}-\mathrm{K}_{2} \mathrm{HPO}_{4}$ and three tie-lines of UCON$\mathrm{NaH}_{2} \mathrm{PO}_{4}$. Further detail on the system composition and phase equilibria were presented before [17]. The partition coefficients for the five NP-monosaccharides (4-nitrophenyl- $\beta$-Dglucopyranoside, 4-nitrophenyl- $\beta$-D-galactopyranoside, 4-nitrophenyl- $\alpha$-D-mannopyranoside and 4-nitrophenyl- $\beta$-Dfucopyranoside) and four proteins (bovine serum albumin, bovine hemoglobin, horse myoglobin and bovine $\alpha$-chymotrypsinogen A) were experimentally determined in the six biphasic systems, except for hemoglobin in tie-line $\mathrm{C}$ of Ucon- $\mathrm{NaH}_{2} \mathrm{PO}_{4}$ ATPS. For this particular case, protein precipitation in the interface was observed and therefore no partition coefficient was determined. The results obtained, together with the feed composition for each tie-line, are presented in Table 1.

In order to investigate the suitability of the Collander equation in polymer-salt ATPS, different correlations were tested using the partition coefficients of the nine solutes determined in this work and also the partition coefficients of five DNP-amino acids previously reported [18] for the same ATPS. Therefore, the partition coefficients for a total of 14 solutes belonging to chemically different families (five DNP-amino acids, five NP-monosaccharides and four proteins) were used for each biphasic system. The only exception is for tie-line $\mathrm{C}$ on Ucon- $\mathrm{NaH}_{2} \mathrm{PO}_{4}$ ATPS, which has only 13 partition coefficients, as explained above.

When the logarithms of the partition coefficients of the solutes in a given tie-line of an ATPS are plotted against the logarithms of the partition coefficients of the solutes in other tie-lines of the same ATPS, straight lines were obtained. This linear correlation is illus- 


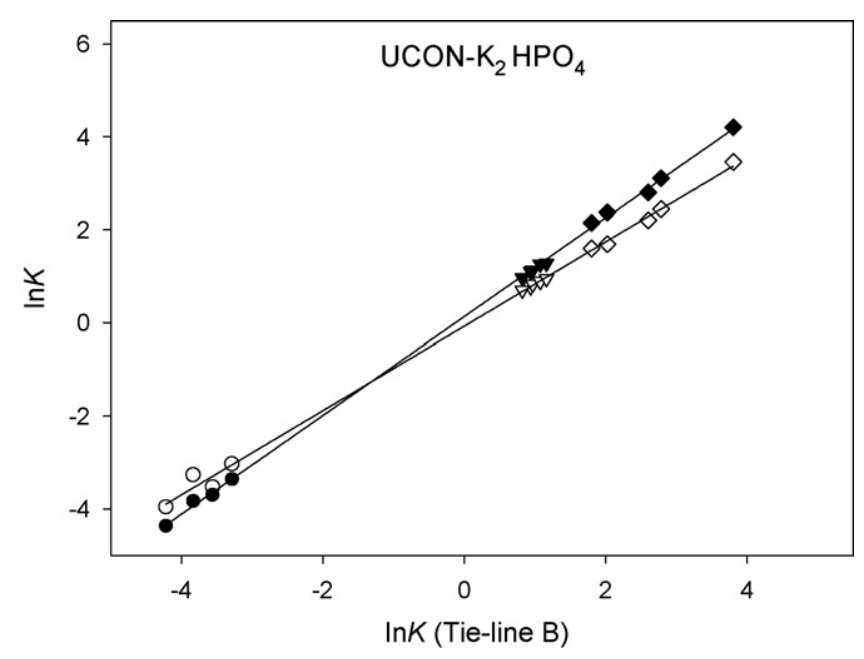

Fig. 1. Correlation between the logarithms of the partition coefficients in different tie-lines of the UCON- $\mathrm{K}_{2} \mathrm{HPO}_{4}$ ATPS, using tie-line B as reference. Tie-line A: $\bigcirc$ proteins, $\nabla$ NP-monossaccharides and $\diamond$ DNP-amino acids; Tie-line C: $\bullet$ proteins, $\mathbf{\nabla}$ $\mathrm{NP}$-monossaccharides and $\diamond \mathrm{DNP}$-amino acids.

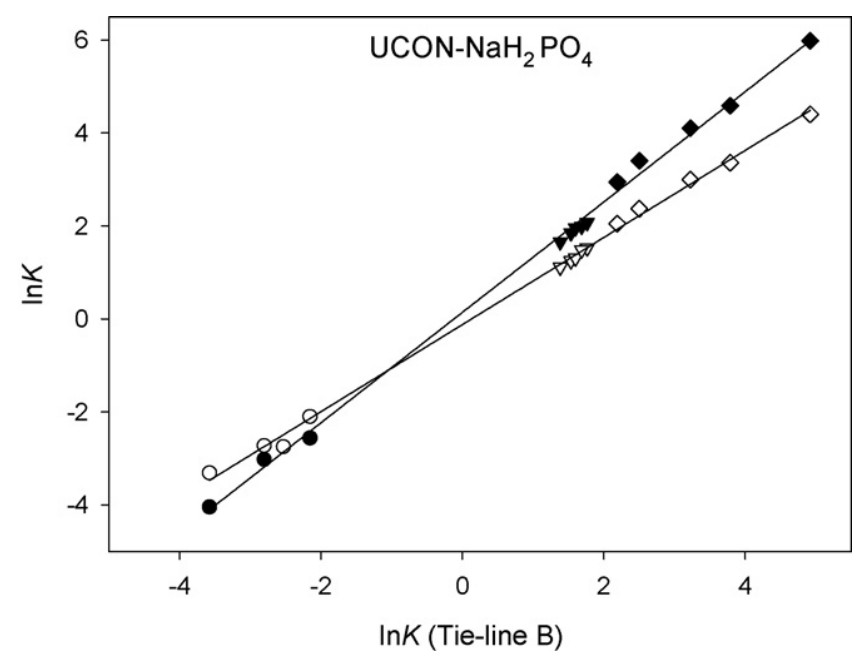

Fig. 2. Correlation between the logarithms of the partition coefficients in different tie-lines of the UCON- $\mathrm{NaH}_{2} \mathrm{PO}_{4}$ ATPS, using tie-line B as reference. Tie-line A: $\bigcirc$ proteins, $\nabla$ NP-monossaccharides and $\diamond$ DNP-amino acids; Tie-line C: $\bullet$ proteins, $\mathbf{\nabla}$ NP-monossaccharides and $\diamond$ DNP-amino acids.

Table 2

Coefficient of determination, $a$ and $b$ values and the statistic parameter $F$ obtained for the representation of the logarithms of the partition coefficients in different tie-lines of the same ATPS.

\begin{tabular}{cccccc}
\hline Tie-lines & $N$ & \multicolumn{2}{l}{$b$} & $r^{2}$ & $F$-statistic \\
\hline UCON- ${ }_{2} \mathrm{HPO}_{4}$ ATPS & & & \\
B-A & 14 & $-0.070 \pm 0.031$ & $0.905 \pm 0.012$ & 0.998 & 5994 \\
B-C & 14 & $0.137 \pm 0.018$ & $1.0609 \pm 0.0070$ & 0.9995 & 23257 \\
UCON-NaH & $\mathrm{PO}_{4}$ & ATPS & & & \\
B-A & 14 & $-0.118 \pm 0.033$ & $0.936 \pm 0.012$ & 0.998 & 5882 \\
B-C & 13 & $0.144 \pm 0.046$ & $1.187 \pm 0.017$ & 0.997 & 4288 \\
\hline
\end{tabular}

trated in Fig. 1 for UCON-K $\mathrm{HPO}_{4}$ and in Fig. 2 for UCON-NaH $\mathrm{HO}_{4}$. The linearity observed is described as:

$\ln K_{\alpha}=a+b \ln K_{\beta}$

where $\alpha$ and $\beta$ correspond to different tie-lines of the same biphasic system and the constants $a$ and $b$ represent the slope of the straight line and the intercept, respectively, obtained from linear regression. Table 2 presents the $a$ and $b$ values, the coefficient of determina-

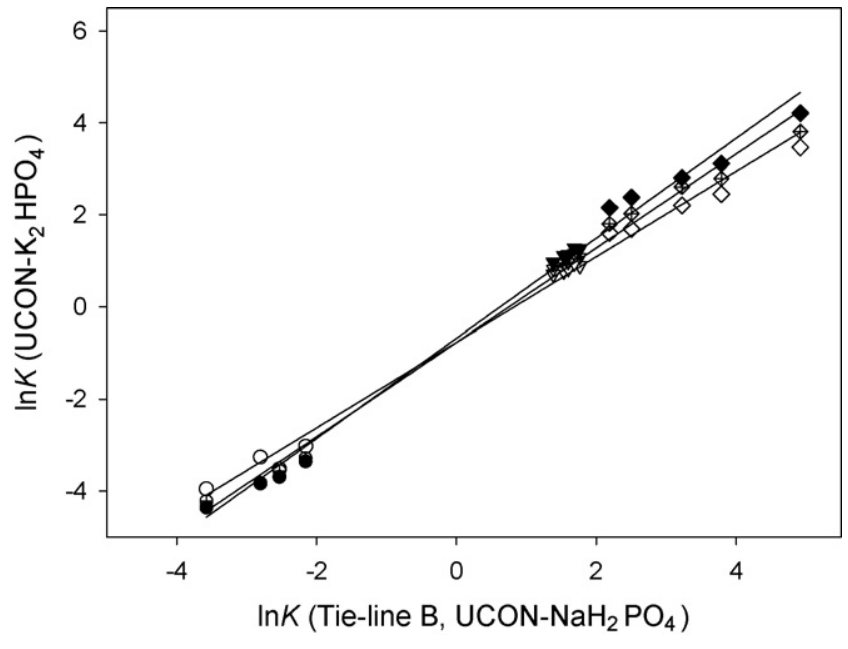

Fig. 3. Correlation between the logarithms of the partition coefficients in UCON$\mathrm{K}_{2} \mathrm{HPO}_{4}$ ATPS, using tie-line B of UCON-NaH $\mathrm{PO}_{4}$ ATPS as reference. Tie-line A: $\bigcirc$ proteins, $\nabla$ NP-monossaccharides and $\diamond$ DNP-amino acids; Tie-line $\mathrm{B}: \oplus$ proteins, $\rightarrow$ NP-monossaccharides and $\bigoplus$ DNP-amino acids; Tie-line C: $\bullet$ proteins, $\nabla$ NPmonossaccharides and $\bullet$ DNP-amino acids.

tion $\left(r^{2}\right)$, the statistic parameter $F$ as well as the total number of experimental points $(N)$ for each correlation represented in Fig. 1 $\left(\mathrm{UCON}-\mathrm{K}_{2} \mathrm{HPO}_{4}\right)$ and Fig. $2\left(\mathrm{UCON}-\mathrm{NaH}_{2} \mathrm{PO}_{4}\right)$. All the lines found present coefficients of determination higher than 0.997 and high $F$ values. In order to prove that the experimental data are correlated by straight lines, the same partition coefficients were correlated according to a second order polynomial:

$\ln K_{\alpha}=a+b \cdot \ln K_{\beta}+c \cdot\left(\ln K_{\beta}\right)^{2}$

where $a, b$ and $c$ are constants. For this case the $r^{2}$ values obtained are almost the same than those from Eq. (3), but the $F$ value reduces at least $50 \%$. This indicates that the new $c$ parameter has no statistical significance and linear regression (Eq. (3)) provides a better correlation.

The linear behaviour observed in Figs. 1 and 2 could be expected: Collander reported [7] that this linear relationship between the logarithms of partition coefficients was found when the systems studied were of similar chemical nature. In his original work, the linear relation for binary systems was held for series of (water + 1alkanols) or (water + dialkyl ethers). It is important to highlight that in the regressions considered above (see Table 2), the components used in the formulation of the ATPS are the same (UCON with either $\mathrm{K}_{2} \mathrm{HPO}_{4}$ or $\mathrm{NaH}_{2} \mathrm{PO}_{4}$ ). The only differences among them are on the composition of the systems. Thus, on the tie-line used to measure the partition coefficient, i.e., the Collander equation is used to correlate different tie-lines of the same ATPS. Therefore, the linear behaviour of the Collander equation follows the conclusions of Collander, as the systems compared are of a similar chemical nature. For that reason, the linear relation obtained indicates that the Collander equation is suitable to correlate and predict the partition coefficients of solutes in the same biphasic system, with different compositions of polymer and salt (different tie-lines of the ATPS).

But can the linear relation be held when the phase-forming components of the ATPS are changed? A plot of the partition coefficients of the solutes in the three tie-lines of an ATPS against the partition coefficients of the same solutes in a tie-line of a different ATPS is presented in Fig. 3 (using tie-line $\mathrm{B}$ of the UCON-NaH $\mathrm{H}_{2} \mathrm{PO}_{4}$ ATPS as a reference) and 4 (using tie-line A of the UCON-K $\mathrm{K}_{2} \mathrm{HPO} 4$ ATPS as a reference). The straight lines represented were described by an equation identical to Eq. (3), but now $\alpha$ and $\beta$ stand for tie-lines of different biphasic systems. In all cases, the coefficients of determination were higher than 0.99 . The $r^{2}, a$ and $b$ values, and the statistic 
Table 3

Coefficient of determination, $a$ and $b$ values and the statistic parameter $F$ obtained for the representation of the logarithms of the partition coefficients in different ATPS.

\begin{tabular}{|c|c|c|c|c|c|}
\hline Tie-lines & $N$ & $a$ & $b$ & $r^{2}$ & F-statistic \\
\hline \multicolumn{6}{|c|}{ UCON-K ${ }_{2} \mathrm{HPO}_{4}$ ATPS; reference: tie-line B UCON-NaH${ }_{2} \mathrm{PO}_{4}$ ATPS } \\
\hline A & 14 & $-0.769 \pm 0.068$ & $0.928 \pm 0.025$ & 0.992 & 1393 \\
\hline B & 14 & $-0.771 \pm 0.074$ & $1.024 \pm 0.027$ & 0.992 & 1426 \\
\hline C & 14 & $-0.682 \pm 0.079$ & $1.086 \pm 0.029$ & 0.992 & 1423 \\
\hline \multicolumn{6}{|c|}{ UCON-NaH${ }_{2} \mathrm{PO}_{4}$ ATPS; reference: tie-line A UCON-K ${ }_{2} \mathrm{HPO}_{4}$ ATPS } \\
\hline A & 14 & $0.659 \pm 0.052$ & $1.003 \pm 0.022$ & 0.995 & 2150 \\
\hline $\mathrm{B}$ & 14 & $0.831 \pm 0.068$ & $1.069 \pm 0.029$ & 0.992 & 1393 \\
\hline C & 13 & $1.080 \pm 0.072$ & $1.299 \pm 0.030$ & 0.993 & 1536 \\
\hline \multicolumn{6}{|c|}{ UCON-NaH${ }_{2} \mathrm{PO}_{4}$ ATPS vs. UCON- $\mathrm{K}_{2} \mathrm{HPO}_{4}$ ATPS } \\
\hline- & 41 & $0.763 \pm 0.045$ & $1.025 \pm 0.018$ & 0.989 & 3354 \\
\hline
\end{tabular}

parameter $F$ are indicated in Table 3, as well as the number of experimental points, $N$, used in each correlation of the $\mathrm{UCON}-\mathrm{K}_{2} \mathrm{HPO}_{4}$ and UCON-NaH $\mathrm{PO}_{4}$ ATPS illustrated in Figs. 3 and 4.

Again, when the partition coefficients are correlated according to a second order polynomial (Eq. (4)), the $r^{2}$ increases but the $F$ value decreases in the range $7-34 \%$. This reduction shows that the linear regression is sufficient for data correlation and that the use of further parameters provides no statistical improvement. The only exception found was for tie-line $\mathrm{C}$ on the UCON-NaH $\mathrm{PO}_{4}$ ATPS, using the tie-line A of UCON- $\mathrm{K}_{2} \mathrm{HPO}_{4}$ ATPS as reference, for which the $F$ value increases about $20 \%$. For this dataset, the use of a third parameter in the correlation improves its quality. Thus, for this case a correlation using a third order polynomial was tested: The $F$ value so obtained was lower than all others, denying statistical significance of the fourth parameter. This statistical analysis indicates that for this particular dataset correlation (tie-line $\mathrm{C}$ on the UCON-NaH $\mathrm{PO}_{4}$ ATPS, using the tie-line A of UCON- $\mathrm{K}_{2} \mathrm{HPO}_{4}$ ATPS as reference) a second order polynomial would provide the best description. Nevertheless, the Collander equation also provided an adequate description, with a coefficient of determination of 0.993 and a $F$ value of the same magnitude of all other correlations in Table 3. Since for all the other correlations indicated in Tables 2 and 3 the linear equation provided the best statistical correlation, it can be concluded that the Collander equation can be used to correlate the partition coefficients in the polymer-salt ATPS used here.

It is important to highlight that both the coefficients of determination and the $F$ values obtained using different ATPS (Table 3 ) are smaller than those obtained within the same ATPS (Table 2). Of

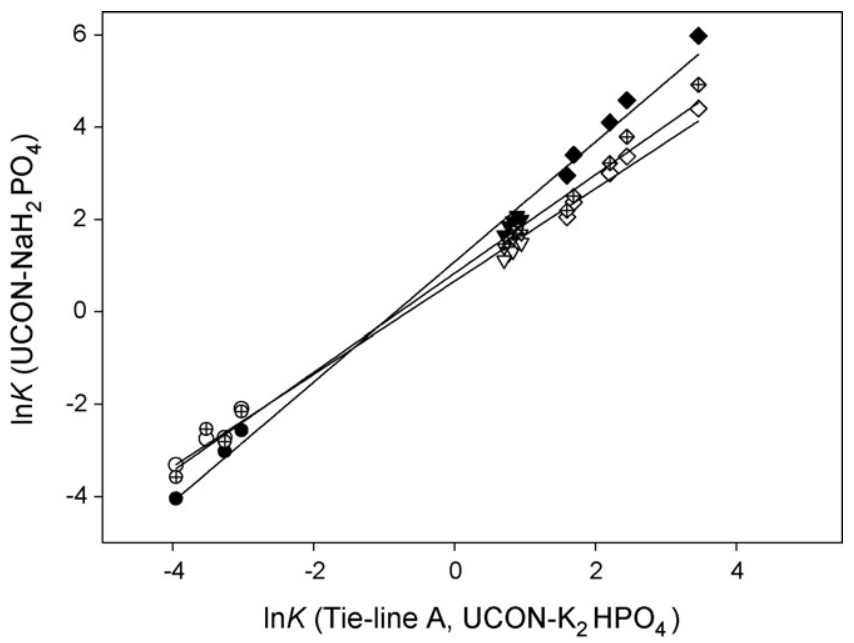

Fig. 4. Correlation between the logarithms of the partition coefficients in UCON$\mathrm{NaH}_{2} \mathrm{PO}_{4}$ ATPS, using tie-line A of UCON-K $\mathrm{K}_{2} \mathrm{HPO}_{4}$ ATPS as reference. Tie-line A: $\bigcirc$ proteins, $\nabla$ NP-monossaccharides and $\diamond$ DNP-amino acids; Tie-line $\mathrm{B}: \oplus$ proteins, $\nabla$ NP-monossaccharides and $\diamond$ DNP-amino acids; Tie-line C: $\bullet$ proteins, $\mathbf{\nabla}$ NPmonossaccharides and $\downarrow$ DNP-amino acids.

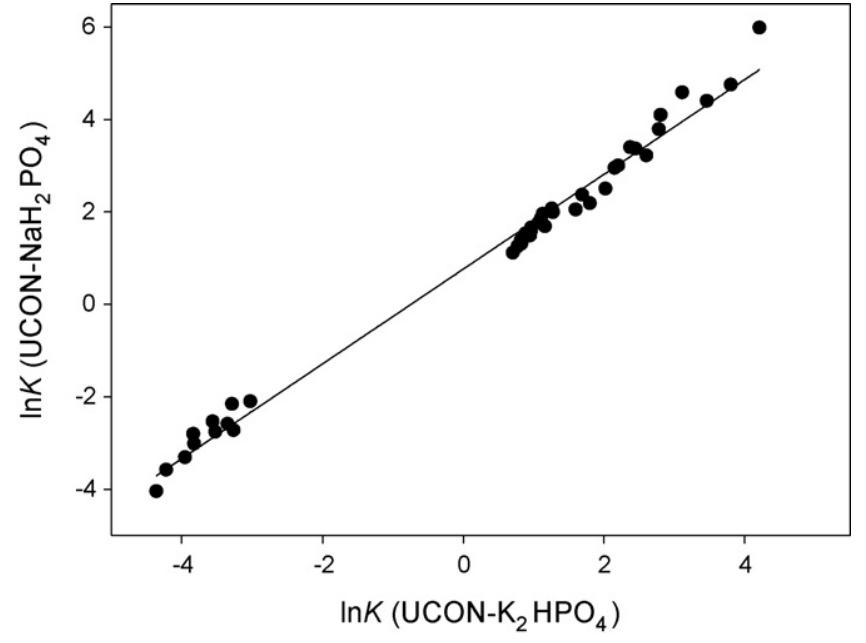

Fig. 5. Correlation between the logarithms of all the partition coefficients in the UCON- $\mathrm{K}_{2} \mathrm{HPO}_{4}$ and the UCON-NaH $\mathrm{PO}_{4}$ ATPS.

course, this effect is due to the change in the type of salt: The ATPS studied here were composed by the same polymer but different salts: $\mathrm{K}_{2} \mathrm{HPO}_{4}$ or $\mathrm{NaH}_{2} \mathrm{PO}_{4}$. This change makes some difference in the nature of the biphasic system, as understood by Collander, and so the linear correlation of partition coefficients is poorer. Still, the quality of the linear regression is satisfactory enough to state that the Collander equation holds for these different polymer-salt ATPS. The results presented, together with previous results obtained in polymer-polymer ATPS [11,12], indicate that the Collander equation is suitable to correlate and predict the partition coefficients of solutes in different ATPS.

Furthermore, the Collander equation was tested for all partition coefficients, independent of the system composition. Thus, partition coefficients in the UCON-NaH $\mathrm{H}_{2} \mathrm{PO}_{4}$ system were plotted as a function of the corresponding partition coefficient in the UCON$\mathrm{K}_{2} \mathrm{HPO}_{4}$ system, and the result is shown in Fig. 5. The results of the linear regression ( $a$ and $b$ values, coefficient of determination, $F$ value and number of experimental points, $N$ ) are presented in Table 3. Again, when a second order polynomial was tested, the $F$ value decreases (ca. 20\%), indicating that just two parameters ( $a$ and $b$ ) are enough for the correlation, and a third parameter would not have statistical significance. The good results obtained for both $r^{2}$ and $F$ indicate that there is a linear relation between all partition coefficients obtained for the systems $\mathrm{UCON}-\mathrm{K}_{2} \mathrm{HPO}_{4}$ and UCON-NaH $\mathrm{PO}_{4}$, independently of the system composition.

\section{Conclusions}

Partition coefficients for different solutes (NP-monosaccharides and proteins) were experimentally determined in three tie-lines of two polymer-salt ATPS: UCON-K $\mathrm{KPO}_{4}$ and $\mathrm{UCON}-\mathrm{NaH}_{2} \mathrm{PO}_{4}$, at 
$23^{\circ} \mathrm{C}$. The partition coefficients for these solutes and others (five DNP-amino acids) obtained in a previous work [18] were used to test the Collander equation. The Collander equation provided a good representation for different tie-lines of the same ATPS. Similar linear relations were obtained between tie-lines of different ATPS, despite the correlation was slightly poorer. Also the correlation using all partition coefficients altogether provided a good representation of the system behaviour. Therefore, the Collander equation can be used to satisfactorily correlate solute partitioning in the studied polymer-salt ATPS. Moreover, the Collander equation can be used for prediction purposes: once the partitioning of a solute in a system is known, its partitioning in other systems can be estimated from the Collander equations. This brings an empirical but rather practical approach for partitioning in ATPS.

\section{Acknowledgements}

Financial support for this work was in part provided by Fundação para a Ciência e a Tecnologia (FCT) and by LSRE financing by FEDER/POCI/2010, for which the authors are thankful. Oscar Rodríguez acknowledges financial support of Programme Ciência 2007 (FCT). Sara C. Silvério acknowledges her Ph.D. grant of FCT (SFRH/BD/43439/2008).

\section{References}

[1] Y. Akama, A. Sali, Talanta 57 (2002) 681-686.

[2] M. Shibukawa, N. Nakayama, T. Hayashi, D. Shibuya, Y. Endo, S. Kawamura, Anal. Chim. Acta 427 (2001) 293-300.

[3] C. Liu, D. Kamei, J. King, D. Wang, D. Blankschtein, J. Chromatogr. B 711 (1998) 127-138.

[4] M. Rito-Palomares, C. Dale, A. Lyddiatt, Biotechnol. Tech. 12 (1998) 711-714

[5] F. Luechau, T.C. Ling, A. Lyddiatt, Sep. Purif. Technol. 68 (2009) 114-118.

[6] R. Hamamoto, M. Kamihira, S. Iijima, J. Ferment. Bioeng. 82 (1996) 73-76.

[7] R. Collander, Acta Physiol. Scand. 13 (1947) 363-381.

[8] R. Battino, F.D. Evans, W.F. Danforth, E. Wilhelm, J. Chem. Thermodyn. 3 (1971) 743-751.

[9] G.G. Briggs, J. Agric. Food Chem. 29 (1981) 1050-1059.

[10] X. Ming, S. Han, Z. Qi, D. Sheng, H. Lian, Talanta 79 (2009) 752-761.

[11] P.P. Madeira, J.A. Teixeira, E.A. Macedo, L.M. Mikheeva, B.Y. Zaslavsky, Fluid Phase Equilib. 267 (2008) 150-157.

[12] P.P. Madeira, J.A. Teixeira, E.A. Macedo, L.M. Mikheeva, B.Y. Zaslavsky, J. Chromatogr. A 1190 (2008) 39-43.

[13] J. Persson, A. Kual, F. Tjerneld, J. Chromatogr. B 743 (2000) 115-126.

[14] M. Pereira, Y. Wu, P. Madeira, A. Venâncio, E. Macedo, J. Teixeira, J. Chem. Eng. Data 49 (2004) 43-47.

[15] O. Rodríguez, S.C. Silvério, P.P. Madeira, J.A. Teixeira, E.A. Macedo, Ind. Eng. Chem. Res. 46 (2007) 8199-8204.

[16] S.C. Silvério, P.P. Madeira, O. Rodríguez, J.A. Teixeira, E.A. Macedo, J. Chem. Eng. Data 53 (2008) 1622-1625.

[17] S.C. Silvério, O. Rodríguez, J.A. Teixeira, E.A. Macedo, J. Chem. Eng. Data 55(2010) 1285-1288.

[18] S.C. Silvério, O. Rodríguez, J.A. Teixeira, E.A. Macedo, J. Chem. Thermodynamics (2010), doi:10.1016/j.jct.2010.04.005. 